\title{
Surgical treatment for functional mitral regurgitation secondary to dilated cardiomyopathy: Current options and future trends
}

Francesco Nicolini, Francesco Maestri, Andrea Agostinelli, Alberto Molardi, Filippo Benassi, Alan Gallingani, Tiziano Gherli

Department of Clinical and Experimental Medicine, Section of Heart Surgery, University of Parma, Parma, Italy

Email: francesco.nicolini@unipr.it

Received 13 January 2013; revised 27 February 2013; accepted 18 March 2013

\begin{abstract}
There is an increasing number of patients with mitral regurgitation secondary to dilated cardiomyopathy. Recent data suggest that mitral regurgitation (MR) can be surgically corrected in heart failure with symptomatic improvements and favourable reverse left ventricular remodeling. However, several questions remain to be answered, regarding the optimal management of functional mitral regurgitation, the correct timing of surgery and the choice of the surgical technique to perform in patients affected by dilated cardiomyopathy. In the setting of ischemic chronic cardiomyopathy, data derived from the recent literature suggest that concomitant severe ischemic MR should be addressed during CABG to improve survival and quality of life. Most surgeons perform concomitant CABG and mitral valve surgery in patients with ischemic chronic cardiomyopathy and moderate to severe MR. In the setting of chronic dilated cardiomyopathy, most clinicians would agree that correction of severe $M R$ in heart failure is warranted, mostly due to a symptomatic benefit and reduction of number of re-hospitalizations. Moreover, reverse ventricular remodeling has been demonstrated with undersized annuloplasty rings and correction of MR: this could lead to improved contractility, reduction in left ventricular end-diastolic and end-systolic volumes, and finally to improved NYHA functional class. Recent large studies suggest that patients undergoing mitral valve repair had improved perioperative survival, shorter length of stay, and improved long-term survival than those undergoing mitral valve replacement because the preservation of the subvalvular apparatus seems to result in superior left ventricular remodelling and in greater improvement in NYHA class. In the near future, data from multi-institutional, randomized prospective trials will help to elucidate many of the questions and concerns regarding repair
\end{abstract}

of severe functional mitral regurgitation. Finally, technology applied to heart surgery is continually evolving and will allow more exciting cellular and novel device therapies for the treatment of functional mitral regurgitation secondary to dilated cardiomyopathy.

Keywords: Mitral Valve Regurgitation; Heart Failure; Cardiac Surgery

\section{INTRODUCTION}

Nearly 6 million Americans suffer from heart failure [1] and many of these patients have a dilated cardiomyopathy based on either ischemic or idiopathic dilated etiology. Functional mitral regurgitation (MR) is a frequent complication of dilated cardiomyopathy and it is related to a dilated annulus with tethered papillary muscles resulting from a dilated, nonelliptical, spherical ventricle, in the presence of a structurally normal valve and subvalvular apparatus. Functional MR worsens the symptoms of chronic heart failure and it has been well demonstrated that it is a significant factor for increased mortality in the natural history of these patients [2].

Although the correction of MR with a simple annuloplasty has been advocated as a safe procedure with acceptable mortality [3,4], it remains a matter of debate whether this could allow improved long-term survival. Other authors supported undersized annuloplasty ring implantation, stressing the concept that a small ring can facilitate reverse remodeling to a more elliptical ventricular shape [5].

Particularly in the setting of chronic ischemic cardiomyopathy, the optimal management of patients with concomitant functional MR remains to be established [6-9]. The benefit of mitral valve plasty (MVP) compared with mitral valve replacement (MVR) has been shown convincingly in patients affected by degenerative mitral 
regurgitation (MR), but such an advantage remains controversial in the presence of chronic ischemic MR [6-14], particularly in case of concurrent left ventricular dysfunction $[15,16]$.

Finally, in consideration of several studies reporting high recurrence of MR, it appears mandatory to discuss the correct indications for the optimal surgical management of functional MR in dilated cardiomyopathies.

\section{MITRAL REGURGITATION SECONDARY TO CHRONIC ISCHEMIC CARDIOMYOPATHY}

Regardless of the specific etiology of heat failure, all patients with left ventricular dysfunction experience remodeling of the ventricle towards a progressive dilation [17]. Normal function of the mitral valve apparatus depends from a correct interaction among the mitral annulus, the leaflets, the sub-valvular apparatus, and the subtending myocardium [18]. It is well known that the papillary muscles are displaced as a consequence of left ventricular dilatation, creating a tension on the mitral leaflets and causing a secondary lack of coaptation. This pattern results in a functional MR in presence of a valve without intrinsic disease. The presence of such a functional MR creates a vicious cycle, worsening left ventricular volume overload, leading to further dilatation and then to a more severe MR.

Particularly in chronic ischemic cardiomyopathy the ventricular dilation tends to be asymmetric depending on the variable areas of myocardial infarction, whereas patients with different etiology of cardiomyopathy present symmetric ventricular dilation [19].

Surgical repair or replacement of the mitral valve can lead to the interruption of this cyclic problem, preventing further ventricular dilation and thus prolonging survival and improving quality of life. The choice to repair or replace the mitral valve concomitant with the coronary revascularization appears reasonable in consideration of greater risk of postoperative death after CABG alone [20]. On the other hand, this decision is controversial because of the retrospective nature of the available scientific results and the lack of evidence of the optimal timing and the correct indications for the correction of functional MR.

Severe MR (4+) associated or not with symptoms of heart failure remains a mandatory indication to surgical correction, independently from the need for coronary revascularization. On the other hand the benefits of treating moderate (3+) MR in the absence of heart failure symptomatology remains a matter of debate.

Three-year survival in patients undergoing percutaneous coronary intervention for acute coronary syndromes with concomitant ischemic MR has been reported to range from $46 \%-76 \%$, on the basis of the severity of
MR [21]. For moderate MR, 5-year survival ranges between $60 \%$ and $70 \%$ [22,23] whereas one-year mortality rates for severe MR are reported as high as 40\% [24]. Moreover, in the setting of coronary artery bypass grafting (CABG), myocardial revascularization alone in patients with chronic ischemic MR has a higher hospital mortality than in patients without valve insufficiency [25]. These data suggest that concomitant severe ischemic MR should be addressed during CABG to improve survival and quality of life.

In the setting of moderate functional MR, several studies have compared the results of CABG alone versus CABG with concomitant MVR [26-30]. Some of them proved no improvement in survival after CABG with concomitant MVR [26,27,29,31] whereas others found a significant improvement in follow-up survival [28,3235]. Although it remains unclear whether there is a survival benefit with repair, it is well known that there are improvements in symptoms, exercise tolerance, and reverse ventricular remodelling [28,32-35]. Regardless of the controversial evidence, there is an increasing trend toward performing MVR at the time of CABG [20]. A prospective, randomized trial, the Moderate Mitral Regurgitation in Patients Undergoing CABG trial [36], is enrolling patients to answer this question. Currently, most surgeons will perform concomitant CABG and MVR in patients with ischemic chronic cardiomyopathy and moderate to severe MR.

As these patients are at even higher risk than those with coronary artery disease and left ventricluar dysfunction, optimal patient selection is mandatory. Factors that have been shown to be predictive of better outcome from MVP include the presence of more viable myocardium, less papillary muscle dyssynchrony (<60 milliseconds), and a shorter mitral valve coaptation depth [20,37]. On the other hand, the presence of preoperative inotropic support and LV end-diastolic diameter greater than 65 $\mathrm{mm}$ have been found to be associated with worse mortality [38].

\section{MITRAL REGURGITATION SECONDARY TO DILATED CARDIOMYOPATHY}

Significant MR occurs in 35\% to 50\% of all patients with chronic heart failure, including nonischemic dilated cardiomyopathy (DCM) [39]. In patients with left ventricular dysfunction, MR results a strong and independent predictor of mortality [40]. As the degree of MR increases, survival rates worse significantly, with a 4year survival in this patient population of $49 \%$ to $57 \%$ [41]. Although MR clearly increases the mortality associated with heart failure, indications for surgical intervention remain a matter of debate because mitral valve surgery is an independent predictor of mortality after 
cardiac surgery [42] and no study has demonstrated that MR correction improves survival in this setting.

Different etiologies of DCM, including idiopathic, viral, postpartum, valvular, and storage diseases causes, can result in functional MR by similar mechanisms as described above for ischemic cardiomyopathy. The anatomical cause of MR in dilated cardiomyopathy is related mainly to the tethering of the mitral valve leaflets because of left ventricular dilatation and change in the geometry from an elliptical to spherical shape [39,43-45]. A loss of contractile function without a change in left ventricular geometry does not result in significant MR [46].

In this setting repair or replacement of the mitral valve prevents ongoing volume overload of the ventricle and thus exacerbation of pathologic remodelling, and alleviates LV wall stress, thereby facilitating a more efficient elliptical geometry. Several isolated, nonrandomized, single-center studies report benefits both from left ventricular remodeling and, in a few reports, survival standpoint $[47,48]$. However, a lack of large, prospective, randomized, multi-institutional data demonstrating the advantage to surgery over medical therapy reduces the consensus on the benefits of surgical correction of MR. In addition, data from several small studies suggest that there is not a survival benefit to surgical correction of MR over medical therapy [49-51].

Most clinicians would agree that correction of severe MR in heart failure is warranted, mostly due to a symptomatic benefit and reduction of the number of re-hospitalizations. Moreover, reverse ventricular remodeling has been demonstrated with undersized annuloplasty rings and correction of MR [52-54]: this could lead to improved contractility, reduction in left ventricular enddiastolic and end-systolic volumes, and finally to improved NYHA functional class.

The best clinical data that exist regarding MVR of severe MR in idiopathic dilated cardiomyopathy derived from the results of the subgroup analysis of the ACORN clinical trial. This trial enrolled 300 patients with dilated cardiomyopathy (mostly with idiopathic etiology) and heart failure symptoms to evaluate the safety and efficacy of the CorCap cardiac support device [55]. This study evaluated two groups: patients who underwent mitral valve surgery and those without, respectively. The mitral valve surgery group contained 193 patients who received mitral valve surgery alone (repair or replacement, control group, $n=102$ ), or mitral valve surgery with implantation of the CorCap Cardiac Support Device (treatment group, $n=91$ ), respectively. Patients who did not need mitral surgery $(n=107)$ were randomized to optimal medical therapy (control, $\mathrm{n}=50$ ) or isolated treatment with the CorCap Device (treatment, $\mathrm{n}=57$ ). The mean LV end-diastolic volume was $69.7 \mathrm{~mm}$, ejec- tion fraction was $23.9 \%$, and peak volume of oxygen utilization $\left(\mathrm{VO}_{2}\right)$ was $14.1 \mathrm{~mL} / \mathrm{kg} / \mathrm{min}$. Short-term and long-term follow-up for up to 5 years have been evaluated. In the subgroup of patients receiving mitral valve surgery, the 30-day survival after surgery was $1.6 \%$. Functionally, significant improvements in NYHA class (2.8 to 2.2), 6-minute walk test (40.4-m increase), Minnesota Living with Heart Failure (MLHF) questionnaire, and peak $\mathrm{VO}_{2}$ were noted at 2 years. In addition, a statistically significant improvement in left ventricular ejection fraction was noted at 5 years. All patients experienced left ventricle reverse remodeling after mitral valve replacement. Of these 193 patients, only 29 were noted to have recurrent MR. Interestingly, there was no difference in survival or degree of reverse remodelling between mitral valve repair and replacement [56-58]. Overall survival was $86.5 \%$ at 1 year, $85.2 \%$ at 2 years, and $70 \%$ at 5 years [55-58]. These data from the ACORN trial demonstrate that mitral valve surgery can be performed safely in patients with advanced heart failure with long-term functional and physiological benefits.

\section{SURGICAL STRATEGIES}

\subsection{Indications}

The current indications regarding mitral valve intervention in patients with severe left ventricular dysfunction are controversial because no prospective randomized trials exist that demonstrate a survival benefit. The current American College of Cardiology/American Heart Association valve disease guidelines recommend mitral valve surgery in patients with advanced heart failure, but only if MV repair or replacement with chordal sparing are options [59]. Thus, MVP or MVR should be performed in patients with severe MR secondary to severe left ventricular dysfunction [39,59].

Even though MVP has been demonstrated to be feasible with limited mortality, patients unlikely to benefit from mitral valve intervention include those with irreversible pulmonary hypertension and chronic advanced right ventricular dysfunction. Ideal operative candidates have a less spherical ventricle, have a less fibrotic myocardium, and demonstrate contractile reserve [39].

Several studies have demonstrated large left ventricular end-diastolic volume (>65 mm), large left ventricle end-systolic volume (>51 mm), large left atrial volume, poor left ventricle sphericity index, and very low ejection fraction as predictors of lack of reverse remodeling after MVP or MVR and hence reduced physiological benefit to the patient $[33,38,60,61]$.

\subsection{Type of Surgery}

One of the questions not completely answered in patients with ventricular dysfunction and MR is the optimal type 
of mitral valve intervention. There are 2 general options: to repair or replace the mitral valve. However, there are many types of repair, without a clear consensus in the literature. Although they did not include patients with heart failure, two large studies suggest that patients undergoing MVP had improved perioperative survival, shorter length of stay, and improved long-term survival than those undergoing MVR [62,63]. Another study showed a similar trend favoring MVP over replacement in terms of 5-year survival as long as the mitral valve coaptation depth was less than $10 \mathrm{~mm}$ [37]. When it exceeds this depth, replacement should be performed taking care to preserve the subvalvular apparatus. The preservation of the subvalvular apparatus seems to result in superior left ventricular remodelling [4]. MVP has also been associated with greater improvement in NYHA functional class [4].

MVP does have a problematic failure rate in functional MR related to lack of reverse remodeling and progressive ventricular dilation [52]. De Bonis et al. [64] reported an MVP failure rate of $9 \%$, although other studies, such as that from the Cleveland Clinic, have demonstrated very high recurrence (30\% - 40\%) of severe (3 4+) MR after annuloplasty as soon as 6 months after surgery [65]. These results are in contrast to the findings by Spoor and Bolling [66] in which minimal recurrent MR was seen up to 4 years after MR with rigid annuloplasty by using a ring that was down-sized by 2 sizes.

Gillinov et al. [15] when comparing MVP versus replacement for functional MR, reported 5-year survival of $58 \%$ for valve repair and 36\% for replacement. However, the MVP group had significantly fewer NYHA class IV patients and less severe MR preoperatively. In the propensity matched poorer risk groups (more severe congestive heart failure, MR, and emergency surgery) and for the group as a whole, there was no difference between repair and replacement, and 5-year survival was uniformly $<50 \%$.

About the question on the optimal surgical strategy for the correction of ischemic MR, recently, an Italian multicenter, 15-year, retrospective, propensity score (PS)matched analysis of a robust patient cohort was designed to elucidate comparative effectiveness of MVP and MVR in association with coronary artery bypass grafting (CABG) and in the presence of left ventricular dysfunction [67]. Careful patient selection was carried out purposefully to focus on a homogeneous patient population, eliminating some common confounding factors characterizing published series on the matter, and to define early and long-term outcomes. Of 1006 patients with chronic ischemic MR and impaired left ventricular function 298 (29.6\%) underwent MVR whereas 708 (70.4\%) received MVP. Propensity scores were calculated and 244 pairs of patients were matched. Early deaths were
3.3\% in MVP versus 5.3\% in MVR (P=NS). Eight-year survival was $81.6 \% \pm 2.8 \%$ and $79.6 \% \pm 4.8 \%(\mathrm{P}=0.42)$, respectively. However, actual freedom from all-cause reoperation and valve-related reoperation were $64.3 \% \pm$ $4.3 \%$ versus $80 \% \pm 4.1 \%$, and $71.3 \% \pm 3.5 \%$ versus 85.5\% \pm 3.9 in MVP and MVR, respectively $(\mathrm{P}<0.001)$. Actual freedom from all valve-related complications was $68.3 \% \pm 3.1 \%$ versus $69.9 \% \pm 3.3 \%$ in MVP and MVP, respectively $(\mathrm{P}=0.78)$. Left ventricular function did not improved significantly, and it was comparable in the 2 groups postoperatively. The authors concluded that MVR is a suitable option for patients with chronic ischemic mitral regurgitation and impaired left ventricular function. It provides better results in terms of freedom from reoperation with comparable valve-related complication rates.

Critiques of the failure after annuloplasty are based on the lack of undersizing (2 - 4 sizes), use of a partial rather than complete ring, and the use of a flexible rather than rigid ring. It has been found that there is an almost 4-fold increase (9.5\% vs $2.5 \%$ ) in recurrence rate of ischemic MR by using a flexible ring as compared with a nonflexible ring in patients with a preoperative ejection fraction $<30 \%$ [66]. Gorman and colleagues have demonstrated that use of a saddle-shaped annuloplasty ring decreases leaflet strain, restores normal annular geometry, and might increase repair durability $[68,69]$. Several studies have attempted to identify patients who will have successful long-term correction of MR after an undersized annuloplasty ring. Identified risk factors include the presence of a single central jet as compared with multiple complex jets, severe leaflet tethering, and excessive coaptation depth (>10 mm) [37,61,70,71]. Careful preoperative assessment of leaflet function is vital in determining whether a patient will have long-term benefit from MV annuloplasty for functional MR.

New annuloplasty rings specifically have been designed for functional MR [66,72-74], characterized by various shapes with the aim to improve the durability of valve repair in this setting.

\section{FUTURE TRENDS}

Several percutaneous strategies for treating functional MR are being investigated. A percutaneous mitral annuloplasty device (CARILLON) has been designed to be inserted into coronary sinus to improve leaflet coaptation. In animal models of ischemic MR, this device has demonstrated to be effective $[75,76]$. Initial data from clinical trials have demonstrated safety and feasibility for the CARILLON device $[77,78]$.

Additional percutaneous devices have attempted to improve valvular competence by using a clip (MitraClip, Abbott Laboratories, Abbott Park, IL) to re-approximate the valve leaflets, much like an Alfieri-type repair. Analysis in a high surgical risk population demonstrates 
feasibility and a hemodynamically significant reduction in MR, although many patients are left with lesser degrees of MR, limiting its widespread use [79,80]. Several percutaneous transcatheter MVR devices are being investigated in large animal models and have shown the potential for translation into clinical therapy. These transcatheter therapies might provide a mean to treat symptomatic MR in patients believed to be at prohibitive risk for traditional surgery.

During recent years, the importance of infarct stabilization in reducing adverse remodelling and subsequent left ventricular dilatation has become recognized. This new approach is based on the use of injectable polymers into the myocardium to stiffen and thicken infarct area in the early post-myocardial infarction period to normalize left ventricular wall stress and attenuate adverse left ventricle remodelling. A lot of biopolymers have been used for this therapeutic purpose, including fibrin, hyaluronic acid, peptides, and thermoresponsive gels. Initial data demonstrate improved wall stress, limited infarct expansion, and reduced global ventricular remodeling after polymer-based infarct stabilization [81].

In the near future, data from multi-institutional, randomized prospective trials will help to elucidate many of the questions and concerns regarding repair of severe functional mitral regurgitation.

It is an exciting time in the management of heart failure because technology applied to heart surgery is continually evolving and will allow more interesting cellular and novel device therapies for the treatment of functional MR secondary to dilated cardiomyopathy.

\section{REFERENCES}

[1] Roger, V.L., Go, A.S., Lloyd-Jones, D.M., et al. (2012) Heart disease and stroke statistics-2012 update: A report from the American Heart Association. Circulation, 125, e2-e220. doi:10.1161/CIR.0b013e31823ac046

[2] Trichon, B.H., Felker, G.M., Shaw, L.K., et al. (2003) Relation of frequency and severity of mitral regurgitation to survival among patients with left ventricular systolic dysfunction and heart failure. American Journal of Cardiology, 91, 538-543.

doi:10.1016/S0002-9149(02)03301-5

[3] Bolling, S.F., Pagani, F.D., Deeb, G.M., et al. (1998) Intermediate-term outcome of mitral reconstruction in cardiomyopathy. The Journal of Thoracic and Cardiovascular Surgery, 115, 381-386. doi:10.1016/S0022-5223(98)70282-X

[4] Bishay, E.S., McCarthy, P.M., Cosgrove, D.M., et al. (2000) Mitral valve surgery in patients with severe left ventricular dysfunction. European Journal Cardio-Thoracic Surgery, 17, 213-221. doi:10.1016/S1010-7940(00)00345-6

[5] Tibayan, F.A., Rodriguez, F., Langer, F., et al. (2004) Undersized mitral annuloplasty alters left ventricular shape during acute ischemic mitral regurgitation. Circulation, 110, II98-I102.

[6] Pierard, L.A., and Carabello, B.A. (2010) Ischaemic mitral regurgitation: Pathophysiology, outcomes and the conundrum of treatment. European Heart Journal, 31, 2996-3005. doi:10.1093/eurheartj/ehq411

[7] LaPar, D.J., and Kron, I.L. (2011) Should all ischemic mitral regurgitation be repaired? When should we replace? Current Opinion in Cardiology, 26, 113-117. doi:10.1097/HCO.0b013e3283439888

[8] Bouma, W., van der Horst, I.C.C., Wijdh-den Hamer, I.J., et al. (2010) Chronic ischemic-based mitral regurgitation: Current treatment results and new mechanism-based surgical approaches. European Journal Cardio-Thoracic Surgery, 37, 170-185. doi:10.1016/j.ejcts.2009.07.008

[9] Borger, M.A., Alam, A., Murphy, P.M., et al. (2006) Chronic ischemic mitral regurgitation: Repair, replace or rethink? The Annals of Thoracic Surgery, 81, 1153-1161. doi:10.1016/j.athoracsur.2005.08.080

[10] Murphy, M.O., Rao, C., Punjabi, P.P., et al. (2011) In patients undergoing mitral surgery for ischaemic mitral regurgitation is it preferable to repair or to replace the mitral valve? Interactive Cardiovascular and Thoracic Surgery, 12, 218-28. doi:10.1510/icvts.2010.245191

[11] Vassileva, C.M., Boley, T., Markwell, S., et al. (2011) Meta-analysis of short-term and long-term survival following repair versus replacement for ischemic mitral regurgitation. European Journal Cardio-Thoracic Surgery, 3, 295-303. doi:10.1016/j.ejcts.2010.06.034

[12] Maltais, S., Schaff, H.V., Daly, R.C., et al. (2011) Mitral regurgitation surgery in patients with ischemic cardiomyopathy and ischemic mitral regurgitation: Factors that influence survival. The Journal of Thoracic and Cardiovascular Surgery, 142, 995-1001. doi:10.1016/j.jtcvs.2011.07.044

[13] Chan, V., Ruel, M. and Mesana, T. (2011) Mitral valve replacement is a viable alternative to mitral valve repair for ischemic mitral regurgitation: A case-matched study. The Annals of Thoracic Surgery, 92, 1358-1366. doi:10.1016/j.athoracsur.2011.05.056

[14] Magne, J., Girerd, N., Senechal, M., et al. (2009) Mitral repair versus replacement for ischemic mitral regurgitation: Comparison of short-term and long-term survival. Circulation, 120, S104-S111. doi:10.1161/CIRCULATIONAHA.108.843995

[15] Gillinov, A.M., Wierup, P.N., Blackstone, E.H., et al. (2001) Is repair preferable to replacement for ischemic mitral regurgitation? The Journal of Thoracic and Cardiovascular Surgery, 122, 1125-1141. doi:10.1067/mtc.2001.116557

[16] Grossi, E.A., Goldberg, J.D., LaPietra, A., et al. (2001) Ischemic mitral valve reconstruction and replacement: Comparison of long-term survival and complications. The Journal of Thoracic and Cardiovascular Surgery, 122, 1107-1124. doi:10.1067/mtc.2001.116945

[17] Mihaljevic, T., Lam, B.K., Rajeswaran, J., et al. (2007) Impact of mitral valve annuloplasty combined with revascularization in patients with functional ischemic mitral regurgitation. Journal of the American College of Cardi- 
ology, 49, 2191-2201. doi:10.1016/j.jacc.2007.02.043

[18] Oliveira, J.V., Vicente, W.V., Rodrigues, A.J., et al. (2009) Mitral insufficiency surgery to treat advanced heart failure. Revista Brasileira de Cirurgia Cardiovascular, 24, 540-551. doi:10.1590/S0102-76382009000500016

[19] Kwan, J., Shiota, T., Agler, D.A., et al. (2003) Geometric differences of the mitral apparatus between ischemic and dilated cardiomyopathy with significant mitral regurgitation: Real-time three-dimensional echocardiography study. Circulation, 107, 1135-1140. doi:10.1161/01.CIR.0000053558.55471.2D

[20] Penicka, M., Linkova, H., Lang, O., et al. (2009) Predictors of improvement of unrepaired moderate ischemic mitral regurgitation in patients undergoing elective isolated coronary artery bypass graft surgery. Circulation, 120, 1474-1481. doi:10.1161/CIRCULATIONAHA.108.842104

[21] Ellis, S.G., Whitlow, P.L., Raymond, R.E., et al. (2002) Impact of mitral regurgitation on long-term survival after percutaneous coronary intervention. American Journal of Cardiology, 89, 315-318.

doi:10.1016/S0002-9149(01)02231-7

[22] Tamaki, N., Kawamoto, M., Tadamura, E., et al. (1995) Prediction of reversible ischemia after revascularization: Perfusion and metabolic studies with positron emission tomography. Circulation, 91, 1697-1705. doi:10.1161/01.CIR.91.6.1697

[23] Schelbert, H.R. (1995) Different roads to the assessment of myocardial viability: Lessons from PET for SPECT. Circulation, 91, 1894-1895. doi:10.1161/01.CIR.91.6.1894

[24] Hickey, M.S., Smith, L.R., Muhlbaier, L.H., et al. (1988) Current prognosis of ischemic mitral regurgitation: Implications for future management. Circulation, 78, I51I59.

[25] Karp, R.B., Mills, N. and Edmunds Jr., L.H. (1989) Coronary artery bypass grafting in the presence of valvular disease. Circulation, 79, I182-I184.

[26] Diodato, M.D., Moon, M.R., Pasque, M.K., et al. (2004) Repair of ischemic mitral regurgitation does not increase mortality or improve long-term survival in patients undergoing coronary artery revascularization: A propensity analysis. The Annals of Thoracic Surgery, 78, 794-799. doi:10.1016/j.athoracsur.2004.03.022

[27] Kim, Y.H., Czer, L.S., Soukiasian, H.J., et al. (2005) Ischemic mitral regurgitation: Revascularization alone versus revascularization and mitral valve repair. The Annals of Thoracic Surgery, 79, 1895-1901. doi:10.1016/j.athoracsur.2004.11.005

[28] Harris, K.M., Sundt 3rd, T.M., Aeppli, D., et al. (2002) Can late survival of patients with moderate ischemic mitral regurgitation be impacted by intervention on the valve? The Annals of Thoracic Surgery, 74, 1468-1475. doi:10.1016/S0003-4975(02)03920-6

[29] Wong, D.R., Agnihotri, A.K., Hung, J.W., et al. (2005) Long-term survival after surgical revascularization for moderate ischemic mitral regurgitation. The Annals of Thoracic Surgery, 80, 570-577. doi:10.1016/j.athoracsur.2005.03.034

[30] Buja, P., Tarantini, G., Del Bianco, F., et al. (2006) Moderate-to-severe ischemic mitral regurgitation and multivessel coronary artery disease: Impact of different treatment on survival and rehospitalization. International Journal of Cardiology, 111, 26-33. doi:10.1016/j.ijcard.2005.06.035

[31] Fattouch, K., Gruccione, F., Zampognaro, R., et al. (2009) POINT: Efficacy of adding mitral valve restrictive annuloplasty to coronary artery bypass grafting in patients with moderate ischemic mitral valve regurgitation-A randomized trial. The Journal of Thoracic and Cardiovascular Surgery, 138, 278-285. doi:10.1016/j.jtcvs.2008.11.010

[32] Prifti, E., Bonacchi, M., Frati, G., et al. (2001) Ischemic mitral valve regurgitation grade II-III: Correction in patients with impaired left ventricular function undergoing simultaneous coronary revascularization. Journal of Heart Valve Disease, 10, 754-762.

[33] Bax, J.J., Braun, J., Somer, S.T., et al. (2004) Restrictive annuloplasty and coronary revascularization in ischemic mitral regurgitation results in reverse left ventricular remodeling. Circulation, 110, II103-II108.

[34] Geidel, S., Lass, M., Schneider, C., et al. (2005) Downsizing of the mitral valve and coronary revascularization in severe ischemic mitral regurgitation results in reverse left ventricular and left atrial remodeling. European Journal Cardio-Thoracic Surgery, 27, 1011-1016. doi:10.1016/j.ejcts.2005.02.025

[35] Badhwar, V. and Bolling, S.F. (2002) Mitral valve surgery in the patient with left ventricular dysfunction. Seminars in Thoracic and Cardiovascular Surgery, 14, 133136. doi:10.1053/stcs.2002.32314

[36] Wierup, P., Egeblad, H., Nielsen, S.L., et al. (2009) Moderate mitral regurgitation in patients undergoing $\mathrm{CABG}-$ The MoMIC trial. Scandinavian Cardiovascular Journal, 43, 50-56. doi:10.1080/14017430802430950

[37] Calafiore, A.M., Gallina, S., Di Mauro, M., et al. (2001) Mitral valve procedure in dilated cardiomyopathy: Repair or replacement? The Annals of Thoracic Surgery, 71, 1146-1152. doi:10.1016/S0003-4975(00)02650-3

[38] Braun, J., van de Veire, N.R., Klautz, R.J., et al. (2008) Restrictive mitral annuloplasty cures ischemic mitral regurgitation and heart failure. The Annals of Thoracic Surgery, 85, 430-436. doi:10.1016/j.athoracsur.2007.08.040

[39] Di Salvo, T.G., Acker, M.A., Dec, G.W., et al. (2010) Mitral valve surgery in advanced heart failure. Journal of the American College of Cardiology, 55, 271-282. doi:10.1016/j.jacc.2009.08.059

[40] Blondheim, D.S., Jacobs, L.E., Kotler, M.N., et al. (1991) Dilated cardiomyopathy with mitral regurgitation: Decreased survival despite a low frequency of left ventricular thrombus. American Heart Journal, 122, 763-771. doi:10.1016/0002-8703(91)90523-K

[41] Agricola, E., Ielasi, A., Oppizzi, M., et al. (2009) Longterm prognosis of medically treated patients with functional mitral regurgitation and left ventricular dysfunction. European Journal of Heart Failure, 11, 581-587. 
doi:10.1093/eurjhf/hfp051

[42] David, T.E. (2007) Outcomes of mitral valve repair for mitral regurgitation due to degenerative disease. Seminars in Thoracic and Cardiovascular Surgery, 19, 116-120. doi:10.1053/j.semtcvs.2007.04.004

[43] Kono, T., Sabbah, H.N., Rosman, H., et al. (1992) Left ventricular shape is the primary determinant of functional mitral regurgitation in heart failure. Journal of the American College of Cardiology, 20, 1594-1598. doi:10.1016/0735-1097(92)90455-V

[44] Sabbah, H.N., Kono, T., Rosman, H., et al. (1992) Left ventricular shape: A factor in the etiology of functional mitral regurgitation in heart failure. American Heart Journal, 123, 961-966. doi:10.1016/0002-8703(92)90703-X

[45] Sabbah, H.N., Kono, T., Stein, P.D., et al. (1992) Left ventricular shape changes during the course of evolving heart failure. American Journal of Physiology, 263, H266$\mathrm{H} 270$.

[46] Otsuji, Y., Handschumacher, M.D., Schwammenthal, E., et al. (1997) Insights from three-dimensional echocardiography into the mechanism of functional mitral regurgitation: Direct in vivo demonstration of altered leaflet tethering geometry. Circulation, 96, 1999-2008. doi:10.1161/01.CIR.96.6.1999

[47] Gillinov, A.M., Mihaljevic, T., Blackstone, E.H., et al. (2008) Should patients with severe degenerative mitral regurgitation delay surgery until symptoms develop? The Annals of Thoracic Surgery, 90, 481-488. doi:10.1016/j.athoracsur.2010.03.101

[48] De Bonis, M., Taramasso, M,, Verzini, A, et al. (2012) Long-term results of mitral repair for functional mitral regurgitation in idiopathic dilated cardiomyopathy. European Journal Cardio-Thoracic Surgery, 42, 640-646. doi:10.1093/ejcts/ezs078

[49] Wu, A.H., Aaronson, K.D., Bolling, S.F., et al. (2005) Impact of mitral valve annuloplasty on mortality risk in patients with mitral regurgitation and left ventricular systolic dysfunction. Journal of the American College of Cardiology, 45, 381-387. doi:10.1016/j.jacc.2004.09.073

[50] Trichon, B.H., Glower, D.D., Shaw, L.K., et al. (2003) Survival after coronary revascularization, with and without mitral valve surgery, in patients with ischemic mitral regurgitation. Circulation, 108, II103-II110. doi:10.1161/01.cir.0000087656.10829.df

[51] Kim, Y.H., Czer, L.S., Soukiasian, H.J., et al. (2005) Ischemic mitral regurgitation: Revascularization alone versus revascularization and mitral valve repair. The Annals of Thoracic Surgery, 79, 1895-1901. doi:10.1016/j.athoracsur.2004.11.005

[52] De Bonis, M., Lapenna, E., Verzini, A., et al. (2008) Recurrence of mitral regurgitation parallels the absence of left ventricular reverse remodeling after mitral repair in advanced dilated cardiomyopathy. The Annals of Thoracic Surgery, 85, 932-939. doi:10.1016/j.athoracsur.2007.11.021

[53] Takeda, K., Taniguchi, K., Shudo, Y., et al. (2010) Mechanism of beneficial effects of restrictive mitral annuloplasty in patients with dilated cardiomyopathy and functional mitral regurgitation. Circulation, 122, S3-S9. doi:10.1161/CIRCULATIONAHA.109.927855

[54] Ten Brinke, E.A., Klautz, R.J., Tulner, S.A., et al. (2010) Clinical and functional effects of restrictive mitral annuloplasty at midterm follow-up in heart failure patients. The Annals of Thoracic Surgery, 90, 1913-1920. doi:10.1016/j.athoracsur.2010.08.010

[55] Acker, M.A., Bolling, S., Shemin, R., et al (2006) Mitral valve surgery in heart failure: Insights from the Acorn clinical trial. The Journal of Thoracic and Cardiovascular Surgery, 132, 568-577. doi:10.1016/j.jtcvs.2006.02.062

[56] Starling, R.C., Jessup, M., Oh, J.K., et al. (2007) Sustained benefits of the CorCap Cardiac Support Device on left ventricular remodeling: Three year follow-up results from the Acorn clinical trial. The Annals of Thoracic Surgery, 84, 1236-1242. doi:10.1016/j.athoracsur.2007.03.096

[57] Mann, D.L., Kubo, S.H., Sabbah, H.N., et al. (2012) Beneficial effects of the CorCap cardiac support device: Five-year results from the Acorn trial. The Journal of Thoracic and Cardiovascular Surgery, 143, 1036-1042. doi:10.1016/j.jtcvs.2011.06.014

[58] Acker, M.A., Jessup, M., Bolling, S.F., et al. (2011) Mitral valve repair in heart failure: Five-year follow-up from the mitral valve replacement stratum of the Acorn randomized trial. The Journal of Thoracic and Cardiovascular Surgery, 42, 569-574. doi:10.1016/j.jtcvs.2010.10.051

[59] Bonow, R.O., Carabello, B.A., Chatterjee, K., et al. (2008) Focused update incorporated into the ACC/AHA 2006 guidelines for the management of patients with valvular heart disease: A report of the American College of Cardiology/American Heart Association Task Force on Practice Guidelines (Writing Committee to revise the 1998 guidelines for the management of patients with valvular heart disease): Endorsed by the Society of Cardiovascular Anesthesiologists, Society for Cardiovascular Angiography and Interventions, and Society of Thoracic Surgeons. Circulation, 118, e523-e661. doi:10.1161/CIRCULATIONAHA.108.190748

[60] Ciarka, A., Braun, J., Delgado, V., et al. (2010) Predictors of mitral regurgitation recurrence in patients with heart failure undergoing mitral valve annuloplasty. American Journal of Cardiology, 106, 395-401. doi:10.1016/j.amjcard.2010.03.042

[61] Lee, A.P., Acker, M., Kubo, S.H., et al. (2009) Mechanisms of recurrent functional mitral regurgitation after mitral valve repair in nonischemic dilated cardiomyopathy: Importance of distal anterior leaflet tethering. Circulation, 119, 2606-2614. doi:10.1161/CIRCULATIONAHA.108.796151

[62] Thourani, V.H., Weintraub, W.S., Guyton, R.A., et al. (2003) Outcomes and long-term survival for patients undergoing mitral valve repair versus replacement: Effect of age and concomitant coronary artery bypass grafting. Circulation, 108, 298-304. doi:10.1161/01.CIR.0000079169.15862.13

[63] Jokinen, J.J., Hippelainen, M.J., Pitkanen, O.A., et al. 
(2007) Mitral valve replacement versus repair: Propensity-adjusted survival and quality of-life analysis. The Annals of Thoracic Surgery, 84, 451-458. doi:10.1016/j.athoracsur.2007.03.058

[64] De Bonis, M., Lapenna, E., La Canna, G., et al. (2005) Mitral valve repair for functional mitral regurgitation in end-stage dilated cardiomyopathy: Role of the "edge-toedge” technique. Circulation, 112, I402-I408.

[65] McGee, E.C., Gillinov, A.M., Blackstone, E.H., et al. (2004) Recurrent mitral regurgitation after annuloplasty for functional ischemic mitral regurgitation. The Journal of Thoracic and Cardiovascular Surgery, 128, 916-924. doi:10.1016/j.jtcvs.2004.07.037

[66] Spoor, M.T., Geltz, A., Bolling, S.F. (2006) Flexible versus nonflexible mitral valve rings for congestive heart failure: Differential durability of repair. Circulation, 114, I67-I71. doi:10.1161/CIRCULATIONAHA.105.001453

[67] Lorusso, R., MD, Gelsomino, S., Vizzardi, E., et al. (2013) Mitral valve repair or replacement for ischemic mitral regurgitation? The Italian study on the treatment of ischemic mitral regurgitation (ISTIMIR). The Journal of Thoracic and Cardiovascular Surgery, 145, 128-139. doi:10.1016/j.jtcvs.2012.09.042

[68] Jimenez, J.H., Liou, S.W., Padala, M., et al. (2007) A saddle-shaped annulus reduces systolic strain on the central region of the mitral valve anterior leaflet. The Journal of Thoracic and Cardiovascular Surgery, 134, 1562-1568. doi:10.1016/j.jtcvs.2007.08.037

[69] Ryan, L.P., Jackson, B.M., Hamamoto, H., et al. (2008) The influence of annuloplasty ring geometry on mitral leaflet curvature. The Annals of Thoracic Surgery, 86, 749-760. doi:10.1016/j.athoracsur.2008.03.079

[70] Alfieri, O. and De Bonis, M. (2010) Mitral valve repair for functional mitral regurgitation: Is annuloplasty alone enough? Current Opinion in Cardiology, 25, 114-118. doi:10.1097/HCO.0b013e328335ffb0

[71] Magne, J., Pibarot, P., Dagenais, F., et al. (2007) Preoperative posterior leaflet angle accurately predicts outcome after restrictive mitral valve annuloplasty for ischemic mitral regurgitation. Circulation, 115, 782-791. doi:10.1161/CIRCULATIONAHA.106.649236

[72] Maisano, F., Redaelli, A., Soncini, M., et al. (2005) An annular prosthesis for the treatment of functional mitral regurgitation: Finite element model analysis of a dog bone-shaped ring prosthesis. The Annals of Thoracic Surgery, 79, 1268-1275. doi:10.1016/j.athoracsur.2004.04.014

[73] Votta, E., Maisano, F., Bolling, S.F., et al. (2007) The
Geoform disease-specific annuloplasty system: A finite element study. The Annals of Thoracic Surgery, 84, 92-101. doi:10.1016/j.athoracsur.2007.03.040

[74] Daimon, M., Fukuda, S., Adams, D.H., et al. (2006) Mitral valve repair with Carpentier-McCarthy Adams IMR ETlogix annuloplasty ring for ischemic mitral regurgitation: Early echocardiographic results from a multi-center study. Circulation, 114, I588-I593. doi:10.1161/CIRCULATIONAHA.105.001347

[75] Daimon, M., Shiota, T., Gillinov, A.M., et al. (2005) Percutaneous mitral valve repair for chronic ischemic mitral regurgitation: A real-time threedimensional echocardiographic study in an ovine model. Circulation, 111, 2183-2189. doi:10.1161/01.CIR.0000163547.03188.AC

[76] Kaye, D.M., Byrne, M., Alferness, C., et al. (2003) Feasibility and short-term efficacy of percutaneous mitral annular reduction for the therapy of heart failure-induced mitral regurgitation. Circulation, 108, 1795-1797. doi:10.1161/01.CIR.0000096051.23734.28

[77] Schofer, J., Siminiak, T., Haude, M., et al. (2009) Percutaneous mitral annuloplasty for functional mitral regurgitation: Results of the CARILLON mitral annuloplasty device European Union study. Circulation, 120, 326-333. doi:10.1161/CIRCULATIONAHA.109.849885

[78] Siminiak, T., Hoppe, U.C., Schofer, J., et al. (2009) Effectiveness and safety of percutaneous coronary sinusbased mitral valve repair in patients with dilated cardiomyopathy (from the AMADEUS trial). American Journal of Cardiology, 104, 565-570. doi:10.1016/j.amjcard.2009.04.021

[79] Whitlow, P.L., Feldman, T., Pedersen, W.R., et al. (2012) Acute and 12-month results with catheter-based mitral valve leaflet repair: The EVEREST II (endovascular valve edge-to-edge repair) high risk study. Journal of the American College of Cardiology, 59, 130-139. doi:10.1016/j.jacc.2011.08.067

[80] Feldman, T., Wasserman, H.S., Herrmann, H.C., et al. (2005) Percutaneous mitral valve repair using the edgeto-edge technique: Six-month results of the EVEREST phase I clinical trial. Journal of the American College of Cardiology, 46, 2134-2140. doi:10.1016/j.jacc.2005.07.065

[81] Dixon, J.A., Gorman, R.C., Stroud, R.E., et al. (2011) Targeted regional injection of biocomposite microspheres alters post-myocardial infarction remodeling and matrix proteolytic pathways. Circulation, 124, S35-S45. doi:10.1161/CIRCULATIONAHA.111.035774 